\title{
Gute Arbeit in Krisenzeiten - Arbeitspolitik zwischen defensiver Krisenabwehr und offensiver Krisenüberwindung
}

\author{
Klaus Pickshaus \\ Hans-Jürgen Urban
}

\begin{abstract}
Die globale Wirtschaftsleistung befindet sich im freien Fall, 2009 dürfte sie erstmalig sinken. Die deutsche Wirtschaft steuert auf die tiefste Rezession der Nachkriegzeit zu. Mit bisher unbekannter Geschwindigkeit brechen der deutschen Export-Wirtschaft Märkte und Aufträge weg. Dem folgt gegenwärtig ein rasanter Rückgang von Produktion und Kapazitätsauslastung. Und durch die drastisch verschlechterten Bedingungen externer Unternehmensfinanzierung sehen sich vor allem die Unternehmen durch Liquiditätsengpässe in ihrer Existenz bedroht, die trotz exorbitanter Gewinne in den vergangenen Jahren keine stabile Eigenkapitalbasis aufgebaut haben. Jetzt rächen sich die Liquiditätsabflüsse an Finanzinvestoren oder die Shareholder-Value-getriebenen maßlosen Dividendenausschüttungen und üppigen Aktienrückkaufprogramme. Sie haben die Unternehmen ihrer ökonomischen Substanz beraubt, die sie nun zum Überleben in der Krise schmerzlich vermissen.
\end{abstract}

\section{EINE „GROßE KRISE“}

Kein Zweifel: Wirtschaftliche Krisen waren noch nie die Stunde „Guter Arbeit“. Nicht selten verliert das Thema humaner Arbeitsbedingungen mit einer rückläufigen wirtschaftlichen Entwicklung an Konjunktur. Das gilt umso mehr für eine rasante ökonomische Abwärtsphase, in der nicht eine kleine Konjunkturkrise, sondern eine mehrdimensionale „große Krise“ eines kapitalistischen Entwicklungsmodells zum Ausdruck kommt. Der Finanzmarkt-Kapitalismus befindet sich in einer Systemkrise, die in nahezu alle Sphären der Ökonomie ausstrahlt.

Das gilt auch für die Unternehmen der Realökonomie. Zwar konnte durch die massive Ausweitung von Kurzarbeit und Qualifizierungsmaßnahmen die drohende Entlassungswelle vorerst abgefangen werden. Doch mit zunehmender Krisendauer werden in den Unternehmen als „Notwehrstrategien “ betitelte Rationalisierungs- und Kostensenkungsprogramme die Oberhand gewinnen. Zu befürchten ist: Die unternehmerischen Restrukturierungsprogramme werden auf tiefe Einschnitte in Beschäftigungs-, Arbeits- und Sozialstandards zielen, um den weitreichenden Auslastungsund Liquiditätsproblemen Rechnung zu tragen. Und sie dürften zugleich versuchen, den relativen gesellschaftlichen Konsens über die Dramatik der Krise als Legitimationsressource für die betrieblichen Abbauprogramme zu nutzen.

Drohende Massenentlassungen, die Aufkündigung bisheriger Einkommensund Sozialstandards sowie umfassende Restrukturierungsmaßnahmen werden für die betrieblichen und gewerkschaftlichen Akteure neue Handlungsparameter erzwingen. Die Auswirkungen der Krise auf Beschäftigung und Arbeitsbedingungen könnten dabei durchaus widersprüchlich ausfallen. Auf der einen Seite werden die individuellen Arbeitsplatzängste und das objektive Risiko des Arbeitsplatzverlustes zunehmen. Und wie immer werden steigende Arbeitslosigkeit und Beschäftigungsunsicherheit die Arbeitsbedingungen massiv unter Druck setzen. Zu erwarten ist, dass unter dem eigentlich verbrauchten Slogan „Hauptsache Arbeit“ die Absenkung von Qualitätsstandards in der Arbeit zum Krisenabwälzungsprogramm wird. Dies könnte nach einer hoffnungsvollen Phase der Revitalisierung gewerkschaftlicher Arbeitspolitik qualitative Arbeitsstandards erneut in die Defensive verbannen.
Zugleich zeichnet sich aber ab, dass gerade in der Krise der arbeitspolitische Problemdruck zunimmt und der Bedarf an Gute-Arbeit-Strategien zur Abwehr neuer Zumutungen wächst. Bereits heute ist eine extreme Segmentierung in der Krisenbetroffenheit der Beschäftigten zu konstatieren. Während etwa die Beschäftigten in der direkten Produktion Kurzarbeit oder gar den Verlust des Arbeitsplatzes zu befürchten haben, sind andere Beschäftigtengruppen, etwa in den Forschungs- und Entwicklungsabteilungen, mit erheblich verschärften Leistungsbedingungen und Forderungen nach ausufernder Mehrarbeit konfrontiert. Der Entwertung lebendiger Arbeit durch Produktionseinschränkungen und Arbeitsplatzabbau steht die intensivere Vernutzung der Arbeit durch Leistungsverdichtung und Mehrarbeit gegenüber.

Diesen Widerspruch spüren die Interessenvertretungen bereits heute: Auch wenn aktuell Gute Arbeit von der TopAgenda verschwindet, herrscht weithin die Wahrnehmung, dass Gesundheit und Arbeitsfähigkeit der Beschäftigten bei einer Verschärfung des Arbeitsdrucks an einem „seidenen Faden “hängen. In den Betrieben könnte sich ereignen, was Peter Bofinger für die Sozialpolitik , am Ende der Krise“ prog-

Klaus Pickshaus, Leiter des Funktionsbereichs Gesundheitsschutz und Arbeitsgestaltung beim Vorstand der IG Metall. e-mail: klaus.pickshaus@igmetall.de Hans-Jürgen Urban, Dr., geschäftsführendes Vorstandsmitglied der IG Metall, zuständig für Sozialpolitik, Gesundheitsschutz und Arbeitsgestaltung. e-mail: hans-juergen.urban@igmetall.de 
nostiziert: dass mit verschärften Cost-Cutting-Strategien, entgrenzten Arbeitszeiten und Leistungsbedingungen, Abbau des Kündigungsschutzes und Beschäftigungsunsicherheiten die „bekannten Rezepte wieder an die Oberfläche gespült werden", die mit in die Krise hineingeführt haben. ${ }^{1}$

\section{DIE TIEFEREN KRISENURSACHEN}

Doch die arbeitspolitischen Fehlentwicklungen, die die Debatte um eine neue Humanisierungsinitiative hervorgebracht haben, reichen weit hinter die gegenwärtige Krise zurück. Ohne eine Analyse der Mechanismen des Finanzmarktkapitalismus und seines Shareholder-Value-Paradigmas wird eine offensive Krisenbewältigung im Interesse der abhängig Beschäftigten nicht $\mathrm{zu}$ entwickeln sein. Dabei ist neben den wirtschafts-, finanz- und sozialpolitischen Deregulierungen, die das Aufblähen der Finanzsphäre beförderten, auch die renditeorientierte Fehlsteuerung in den Unternehmen selbst zu thematisieren. Die Orientierung an immer maßloseren Renditezielen hat in den Unternehmen einen Steuerungs- und Kontrollmodus etabliert, der nicht nur eine Ökonomie der kurzen Fristen, sondern auch eine Maßlosigkeit der Anforderungen in der Arbeit zur Folge hatte.

Diese Strategie hat sich als Sackgasse erwiesen. Die überfällige Überwindung tayloristischer Arbeitsorganisation durch eine systematische Aufwertung der lebendigen Arbeit blieb in einem Amalgan von Marktsteuerung, aus Renditevorgaben abgeleiteten Zielvorgaben und Re-Taylorisierung stecken. Kalmbach und Schumann resümieren: „In der vom Finanzmarkt dominierten Denkweise und dem daraus abgeleiteten Shareholder-Value-Konzept und seiner Kurzfristökonomie ist eine systematische Vernachlässigung der Human-Ressourcen erfolgt".2

In die Sackgasse führte diese Strategie auch, weil sie einem Unternehmensbild folgte, das die Verteilung, und nicht die nachhaltige Sicherung der Wertschöpfung, ins Zentrum des Managementhandelns stellt. Während der Druck auf Einkommen und Arbeitsbedingungen anstieg und betriebliche Restrukturierungsprozesse immer neue Cost-Cutting-Runden eröffneten, sackte der Anteil der Investitionen an den verwendeten Gewinnen ab. Mit steigenden Renditen wurden Investoren bedient, die weniger Zukunftsvorsorge für die
Unternehmen als vielmehr die Akkumulation auf den Finanzmärkten vorantrieben.

\section{GRENZEN DES GESCHEITERTEN MODELLS}

Auch wenn die einseitige Finanzmarktorientierung der Corporate Governance mittlerweile gründlich diskreditiert erscheint, steht eine kritische Aufarbeitung der fatalen unternehmenspolitischen und gesellschaftlichen Fehlsteuerungen nach wie vor aus. Dabei dürften die tiefer liegenden Krisenursachen weniger im individuellen Versagen einzelner Manager, als vielmehr darin zu finden sein, dass sich die Akteure entsprechend den Anforderungen des Finanzmarktkapitalismus rational verhalten haben. Deutlich wird, dass die Steuerungslogik des Shareholder-ValueModells in mehrfacher Hinsicht insbesondere Innovationsprozesse blockiert:

(1) Eine Leistungssteuerung, die fast ausschließlich auf betriebswirtschaftlichen, an Kostenzielen orientierten Kennziffern basiert, negiert die tatsächliche Leistungsfähigkeit von Mensch und Maschine und ignoriert zumeist auch die spezifischen Bedingungen der örtlichen Arbeitsorganisation. Widersprüche zwischen einem ausschließlich an der Verwertungslogik orientierten Handeln einerseits und den zu lösenden Problemen der Qualität der Produkte und Verfahren andererseits, werden zuhauf berichtet.

(2) Hinzu kommt, dass die kurzatmige Handlungslogik der Shareholder-Ökonomie den Mut zur Innovation schwächt, deren Erfolg sich ja in der Regel erst mittelund langfristig zeigt. Der Verlust an Planungshorizonten und Sicherheiten befördert zudem bei den Beschäftigten als den eigentlichen Innovationsträgern Demotivation und Angst.

(3) Schließlich werden systematisch unrealistisch hohe Zielvorgaben produziert, die real nicht umsetzbar sind und $\mathrm{zu}$ einer chronischen Überforderung der Beschäftigten beitragen. Zahlreiche wissenschaftliche Untersuchungen weisen auf die Grenzen der Belastbarkeit der Beschäftigten hin und warnen, dass eine Verstärkung des Leistungsdrucks und eine weitere Verdichtung der Arbeit zu einer Zermürbung des Arbeitsvermögens und $\mathrm{zu}$ verheerenden gesundheitlichen Folgen führen könnten. ${ }^{3}$
Umgekehrt gibt es viele Hinweise für ein neues Anspruchsniveau zahlreicher Beschäftigter, die auch unter dem Krisendruck und angesichts der Arbeitsplatzbedrohung das Thema der Qualität der Arbeits- und Leistungsbedingungen nicht ad acta legen wollen.

\section{REAKTIVE KRISENABWEHR ODER OFFENSIVE KRISENBEWÄLTIGUNG}

Angesichts dieser Ausgangslage ist darauf zu beharren, dass auch unter Krisenbedingungen divergierende arbeitspolitische Weichenstellungen denkbar und möglich sind. Krisen können durch eine Schockstarre einen "Tunnelblick“ befördern. Zweifelsohne stehen die Gewerkschaften angesichts der drohenden Verluste an Mitgliedern und Organisationsmacht sowie der Komplexität einer erfolgreichen Krisenabwehr objektiv vor einem Überforderungsproblem. Dies könnte eine Verengung der Aktionsfelder auf das sogenannte "gewerkschaftliche Kerngeschäft" befördern. Unter dem Druck auf Arbeitsplätze und Einkommen rücken die Sicherung der Beschäftigung und der Widerstand gegen die Abwälzung der Krisenlasten auf die Beschäftigten in den Vordergrund. Die Errichtung von Schutzdämmen zur Verhinderung der Überwälzung der Krisenlasten auf die abhängig Beschäftigten wird zum zentralen Fundament gewerkschaftlicher Politik in der Krise.

Dennoch: Wenn die offensichtliche De-Legitimierung des Shareholder-ValueKonzeptes als Ausgangspunkt eines arbeitspolitischen Paradigmenwechsels genutzt werden soll, müssen offensivere und breitere Handlungsansätze entwickelt werden. Krisenhafte Umbrüche sind immer auch ergebnisoffene Phasen einer vertieften Auseinandersetzung um zukunftsfähige Entwicklungspfade. Der mit der Krise verbundene Strukturwandel dürfte auch die Option bieten, Arbeits- und Geschäfts-

1 Bofinger, P. (2009): Für eine neue Balance von Staat und Markt, in: Frankfurter Rundschau v. 7.4., S. 26.

2 Kalmbach, P./Schumann, M. (2008): Finanzkrise als Schocktherapie, in: WSI-Mitteilungen 11+12, S. 637.

3 Vgl. hierzu die diversen Studien, deren Ergebnisse im Jahrbuch Gute Arbeit (Schröder, L./Urban, H.-J. (Hrsg.) (2009): Gute Arbeit. Handlungsfelder für Betriebe, Politik und Gewerkschaften, Frankfurt) und fortlaufend in der Zeitschrift Gute Arbeit berichtet werden. 
prozesse entlang neuer Produkte und $\mathrm{Zu}$ lieferketten, die sich herausbilden werden, neu zu konturieren. Es könnte sich mitten in der Krise für eine gewisse Übergangszeit ein Gestaltungsfenster öffnen, das Ausblicke auf neue arbeitspolitische Weichenstellungen erlaubt. Insofern ist die Debatte um das Ausloten der Chancen für einen neuen arbeitspolitischen Pfad von höchster Aktualität - einen Pfad hin zu arbeitskraftzentrierten und innovationsfördernden Varianten der Arbeitsgestaltung.

Bei der Abwehr schlechter Arbeit, also entgrenzter Arbeitszeiten und Leistungsbedingungen sowie reduzierter Beschäftigungssicherheit, können Themen wie Schutz der Gesundheit und Erhalt der Arbeitsfähigkeit eine zusätzliche Mobilisierungskraft entfalten. Dies gilt insbesondere für die Handlungsfelder der Arbeitszeitund Leistungspolitik sowie Arbeitsgestaltung. Es ist absehbar, dass der Druck auf Kranke und Leistungsgeminderte unter den gegenwärtigen Konstellationen weiter zunehmen wird, bis hin zur Gefahr der Entlassungen.

Schon jetzt erwartet nur jeder zweite Beschäftigte, unter den derzeitigen Arbeitsbedingungen seine Tätigkeit bis zum Rentenalter ausüben zu können. Ein Drittel bezweifelt dies nach der repräsentativen Erhebung des DGB-Index Gute Arbeit ausdrücklich. ${ }^{4}$ In dieser Situation trägt die gesetzliche Beschlussfassung zur Rente mit 67 und zum gleichzeitigen Wegfall der gesetzlich geförderten Altersteilzeit erheblich zur Verunsicherung bei und verhindert in dieser zugespitzten Krisensituation gleichzeitig ein flexibles und sozialverträgliches Ausscheiden der rentennahen Jahrgänge aus den Betrieben, um Arbeitsplätze für jüngere Beschäftigte zu sichern. Die Arbeitszeitpolitik wird wie die Leistungspolitik mit den Anforderungen an eine Entdichtung der Arbeit und Begrenzung der Leistungsintensivierung zu einem wichtigen Kampffeld, auf dem um einen nachhaltigeren Umgang mit der lebendigen Arbeit und damit um die Eröffnung eines neuen arbeitspolitischen Weges gestritten werden muss.

\section{INNOVATION UND PFADE GUTER ARBEIT}

Ein wesentliches Element einer offensiven arbeitspolitischen Krisenbewältigung dürfte darin bestehen, den drohenden radikalisierten Cost-Cutting-Strategien einen in- novationsorientierten Strategieansatz entgegenzusetzen. ${ }^{5} \mathrm{Da}$ Innovationen sich erst über längere Fristen rechnen, setzt eine solche Option schon die Auseinandersetzung mit den gescheiterten Imperativen einer Kurzfristökonomie voraus. Die Stärkung der Innovationsfähigkeit als Teil eines Krisenüberwindungskonzepts wird dabei Innovation in all ihren Dimensionen umfassen müssen: Produktinnovation, Prozessinnovationen und soziale Innovationen.

Der innovationsorientierte Strategieansatz setzt auf Vereinbarkeit von Wirtschaftlichkeit und Sozialverträglichkeit und versucht, betriebliche Wettbewerbsfähigkeit und gute Arbeitsbedingungen gleichermaßen zu realisieren. Der Anspruch, effizienter werdende Wertschöpfungsprozesse mit den arbeitspolitischen Interessen der Beschäftigten in Übereinstimmung zu bringen, macht zweifelsohne seine wirtschaftliche und politische Attraktivität aus. Gleichwohl darf die „janusköpfige Wirkung" (Michael Schumann) eben auch innovativer Produktionskonzepte nicht übersehen werden. Auch innovative Modelle leistungspolitischer und arbeitsorganisatorischer Rationalisierung sind vielfach mit systematischen Interessenverletzungen der Beschäftigten verbunden. Die Entgrenzung von Leistungsanforderungen und Arbeitszeiten, die Verletzung von Beschäftigteninteressen im Rahmen indirekter Personalsteuerungskonzepte und die Segmentierung und machtpolitische Schwächung der Belegschaften gehen vielfach auch mit neuen Produktionskonzepten einher. Auch innovative Arbeit kann schlechte Arbeit sein.

Die innovations- und wettbewerbsorientierte Strategie sollte daher zu einem neuen arbeitskraftzentrierten Ansatz der Arbeitspolitik weiterentwickelt werden. Nicht die Verbesserung der betrieblichen Wettbewerbsfähigkeit durch Kostensenkung oder Innovationen, sondern die Protektion und Profilierung der Interessen der abhängigen Arbeit stellen den strategischen Ansatz dar. Betriebliche Wettbewerbsfähigkeit wird - zumal unter Krisenbedingungen - als Überlebensbedingung auf profitgesteuerten Märkten anerkannt, jedoch nicht zum zentralen Strategieziel erhoben. Stattdessen rücken die Arbeitskraft- und Subjektinteressen der Beschäftigten und die Stärkung von Humanisierungsstandards ins Zentrum. Schlüsselakteure dieses Ansatzes sind Beschäftigte, betriebliche Interessenvertretungen und Gewerkschaften, die auf die Durchsetzung arbeitspolitischer
Standards über Gegenmachtentfaltung setzen. Eigene innovative Gestaltungskonzepte sind in dieser Strategie unverzichtbare Bestandteile einer arbeitsorientierten Interessenpolitik. Und da die arbeitspolitischen Gestaltungsspielräume umso größer sind, je weniger der externe Wettbewerbsdruck betriebliche Handlungsspielräume einengt, gehören wettbewerbsdämpfende Regulierungen auf branchen- und strukturpolitischer Ebene zu einer solchen Strategie.

\section{STRATEGIEZIEL: DEMOKRATISCHE ARBEIT}

In einem solchen arbeitskraftzentrierten Ansatz ist Demokratie in der Arbeit eine essenzielle Voraussetzung für eine erfolgreiche Krisenüberwindungsstrategie. Facetten und Einzelfragen, die sich im weiteren Sinne mit dem Thema demokratische Arbeit befassen, tauchen etwa in der Debatte um direkte Partizipation der Beschäftigten an der Gestaltung ihrer unmittelbaren Arbeits- und Leistungsbedingungen, in der Diskussion um die Sicherung der betrieblichen wie der Unternehmensmitbestimmung und nicht zuletzt in der Debatte um die Perspektive einer Demokratisierung der Finanzökonomie auf. Gleichwohl fügen sich diese Facetten nicht zu einem Gesamtbild und einer Realisierungsstrategie zusammen.

Diese Diskussionsstränge stellen geeignete Anknüpfungspunkte einer neuen Debatte über die Perspektiven demokratischer Arbeit dar. Zweifelsohne kann sinnvoll an die Debatten über Mitbestimmung am Arbeitsplatz oder Wirtschaftsdemokratie angeknüpft werden. Gleichwohl werden Analysen und Strategieempfehlungen aus diesen Debatten angesichts der grundlegend veränderten Realität schnell an Grenzen stoßen. Deswegen gilt es, ein Leitbild demokratischer Arbeit im globalisierten Kapitalismus $\mathrm{zu}$ formulieren und an angemessenen Umsetzungsstrategien zu arbeiten. Eine solche Konzeption - dies könnte

$4 \quad$ Vgl. zu den Ergebnissen der Sonderauswertung des DGB-Index Gute Arbeit: „Arbeitsfähig bis zur Rente?" , Zeitschrift Gute Arbeit 3/2009 mit mehreren Beiträgen.

5 Zum Vorschlag einer Typologie unterschiedlicher arbeitspolitischer Strategieansätze vgl. Pickshaus, K./Urban, H.-J. (2009): Gute Arbeit als Strategie, in: Schröder, L./Urban, H.-J. (Hrsg.): Gute Arbeit, Frankfurt, a.a. O., S. 103ff. 
ein Zwischenergebnis der bisherigen Debatte sein - muss als eine MehrebenenKonzeption formuliert werden. Das bedeutet: Eine solche Konzeption muss die direkte Arbeitsumwelt des Einzelnen, das gesamte Unternehmen sowie die Arenen der Wirtschaftspolitik als Räume einer Demokratisierung von Arbeit wahrnehmen; und sie muss die Einzelforderung für diese Räume zu einer Gesamtkonzeption kombinieren und über die Wechselwirkungen der Entwicklungen in den unterschiedlichen Ebenen nachdenken. Demokratische Ar- beit erfordert Demokratiepolitik am Arbeitsplatz, im Unternehmen und in der Gesellschaft.

Nicht zuletzt die Realisierungsvoraussetzungen demokratischer Arbeit machen deutlich, dass eine arbeitskraftzentriertinnovative Arbeitspolitik über die Arenen des Betriebs hinaus auf die Branchen-, Struktur- und Regionalpolitik erweitert werden muss. Der von der IG Metall vorgeschlagene Beteiligungsfonds zur Unternehmenssicherung könnte einen Weg der öffentlich finanzierten und kontrollierten In- vestitionssteuerung eröffnen, auf dem auch die arbeitspolitischen Entwicklungspfade beeinflusst werden können. ${ }^{6}$ Ohne eine Stärkung solcher wirtschaftsdemokratischen Einflussmöglichkeiten ist eine grundlegende Abkehr vom finanzmarktdominierten Typus der Unternehmens- und Wirtschaftspolitik schwer vorstellbar.

6 Vgl. IG Metall Vorstand (2009): Aktiv aus der Krise - Gemeinsam für ein Gutes Leben. Aktionsplan der IG Metall, Frankfurt, März, download: www.igmetall.de.

\section{Schuldenfalle Hartz IV}

\section{ÜBERSCHULDUNG - EIN VERMITTLUNGSHEMMNIS}

Wer überschuldet ist, tut sich meist sehr schwer damit, einen Arbeitsplatz zu finden. Dies liegt nicht nur an dem bei Überschuldeten häufig zu beobachtenden geringen Selbstbewusstsein, sondern auch daran, dass viele Arbeitgeber mit überschuldeten Mitarbeitern schlicht nichts zu tun haben wollen. Die erste Hürde, an der viele bereits scheitern, ist der bei Bewerbungen übliche Fragebogen. Hier findet sich oftmals die Frage, ob Lohnpfändungen zu erwarten seien. Auch in Vorstellungsgesprächen wird diese Frage gerne gestellt. Da die meisten Menschen viel ehrlicher sind als oft unterstellt wird, beantworten sie eine solche Frage meist wahrheitsgemäß - mit der Folge, dass eine Einstellung in der Regel nicht erfolgt.

Doch auch über denjenigen, die das Glück haben, ein Bewerbungsverfahren erfolgreich zu bestehen, ohne dass ihre Überschuldung thematisiert worden ist, hängt weiterhin ein Damoklesschwert: die Lohnpfändung, die sie den Arbeitsplatz kosten kann. Zwar ist eine Lohnpfändung - von Ausnahmen abgesehen - kein zulässiger Kündigungsgrund; da jedoch ein Arbeitgeber während der Probezeit ohne Angabe von Gründen kündigen kann und es keinerlei gesetzliche Verpflichtung gibt, befristete Arbeitsverträge zu verlängern, können Lohnpfändungen in der Praxis letztlich zum Verlust des Arbeitsplatzes führen. Grund dafür ist zum einen der Aufwand, der bei Lohnpfändungen auf den Arbeitgeber zukommt. Mit Eingang der Lohnpfändung wird er dem Gläubiger seines Arbeitnehmers gegenüber haftbar, den pfändbaren Teil des Lohnes an diesen abzuführen. Da die Pfändungsregeln der Zivilprozessordnung (ZPO) ein Musterbeispiel von vorsichtig ausgedrückt - gesetzlicher Komplexität sind, gerät jeder Arbeitgeber, der sich in dieser Materie nicht sehr genau auskennt, in die Gefahr, Fehler zu machen, für die er unter Umständen dem Gläubiger gegenüber geradestehen muss. Da die meisten Überschuldeten nicht nur einen, sondern mehrere Gläubiger haben, gibt es Arbeitnehmer mit einer ganzen Reihe von Lohnpfändungen, was insbesondere kleine Betriebe, denen es an Erfahrung mit dieser Materie fehlt, viel und für sie unproduktive Arbeitszeit kostet.

Das Stigma der Lohnpfändung kann weitere Schwierigkeiten mit sich bringen. Arbeitnehmerinnen und Arbeitnehmer, die mit viel Bargeld zu tun haben, wie zum Beispiel Kassiererinnen oder Bankangestellte, können unter Umständen, wenn eine Lohnpfändung eingeht, auch nach der Probezeit ihren Arbeitsplatz verlieren. Oder sie werden, falls tatsächlich einmal Geld fehlt, als erste verdächtigt, in die Kasse gegriffen zu haben. In kleineren Betrieben, in denen sich eine Lohnpfändung schnell herumspricht, werden überschul- dete Mitarbeiterinnen und Mitarbeiter auch oft gemobbt, da es als Makel gilt, sein Finanzgebaren nicht im Griff zu haben.

Dass Überschuldung ein Vermittlungshemmnis in Arbeit ist und sich durch eine entsprechende Beratung meist in den Griff bekommen lässt, hat inzwischen auch der Gesetzgeber erkannt. So wurde im Sozialgesetzbuch II (SGB II), besser bekannt als „Hartz IV“, die Schuldnerberatung als sogenannte Eingliederungsleistung definiert ( $\$ 16$, Abs. 2 SGB II). Auf den ersten Blick wirkt Hartz IV in diesem Punkt sehr vernünftig, gibt es doch viele positive Beispiele von ehemals langzeitarbeitslosen Menschen, die während oder nach ihrer Beratung durch eine Schuldnerberatungsstelle einen Arbeitsplatz gefunden haben. Auf den zweiten Blick erweist sich Hartz IV jedoch als moderne Version der aus der griechischen Mythologie bekannten Hydra, jenem Schlangenwesen, dem, wenn ihm ein Kopf abgeschlagen wurde, zwei Köpfe nachwuchsen. Prosaischer ausgedrückt: Hartz IV selbst ist inzwischen zur Schuldenfalle geworden.

Martin Staiger ist evangelischer Theologe und Sozialarbeiter, er arbeitet als Schuldnerberater, Referent und freier Journalist, u.a. für die Blätter für deutsche und internationale Politik (Berlin) und für Publik-Forum (Oberursel).

e-mail: majo.staiger@web.de 